
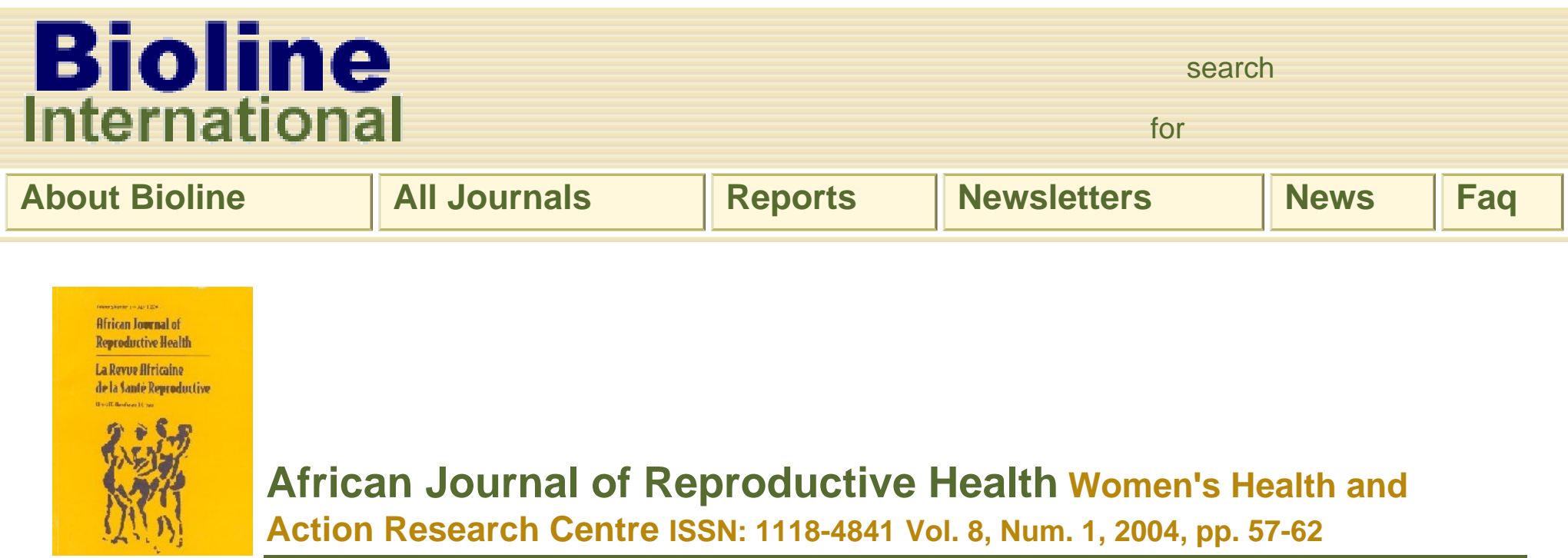

African Journal of Reproductive Health Women's Health and Action Research Centre ISSN: 1118-4841 Vol. 8, Num. 1, 2004, pp. 57-62

African Journal of Reproductive Health, Vol. 8, No. 1, April, 2004 pp. 57-62

\title{
Reducing Maternal Mortality from Unsafe Abortion among Adolescents in Africa
}

Peju Olukoya

Correspondence: Peju Olukoya, Medical Officer/Responsible Officer FCH/Adolescent Sexual and Reproductive Health, Department of Child and Adolescent Health and Development, World Health Organization, 20 Avenue Appia, Switzerland. Tel: 41-22-791-3306; Fax: 41-22-791-4853; E-mail: olukoyaa@who.int

\section{Code Number: rh04010}

\section{Abstract}

Initiation of sexual behaviour is a normal part of human development and it often occurs during adolescence. This is common and universal to all societies and cultures around the world with profound implications for sexual and reproductive health. Adolescence is the period when much of the changes that are associated with becoming an adult take place. These are changes which societies expect and welcome. Unsafe abortion is a major cause of maternal mortality among adolescents in Africa. The need for a public health response to unsafe abortion in Africa is compelling. It is important to focus on primary prevention including the provision of appropriate sexuality education and information as well as supportive services to allow adolescents to prevent unwanted pregnancy. Secondary prevention efforts include prompt diagnosis and treatment of complications by ensuring that services are made more responsive to the needs of adolescents, and by improving access to and quality of care for them. Postabortion care in adolescents must include contraception as well as several elements of life planning. (Afr J Reprod Health 2004; 8[1]:57-62) 
Key Words: Adolescents, unsafe abortions, Africa, prevention, clinical management

\section{Résumé}

Réduction de la mortalitée maternelle occasionée par l'avortement à risque parmi les adolescentes en Afrique. Le déclenchement de comportement sexuel constitue une partie normale du développement humain et il se produit souvent pendant l’adolescence. Ceci est commun et universel à toutes les societés et cultures partout dans le monde, ayant des implications profondes pour la santé sexuelle et de reproduction. L'adolescence est la période où une grande partie des changements liés à la transition en âge adulte ont lieu. Ce sont des changements auxquels la société s`attend et fait bon accueil. L'avortement à risque est une cause importante de la mortalité maternelle parmi des adolescentes en Afrique. La nécessité d`une réponse de la santé publique à l’avortement à risque est irrefutable. II est important de concentrer sur la prévention primaire y compris l’assurance d`une éducation et information sexuelle appropriées comme services de soutien pour permettre aux adolescentes d`éviter la grossesse non-désirées. Les efforts de la prévention secondaire comprennent le diagnostic et le traitement prompts des complications en assurant que des services deviennent plus sensibles aux besoins des adolescentes et en améliorant l’accès au soin de qualité à leur profit. Le soin post-avortement pour les adolescentes doit comprendre la contraception et plusieurs autres éléments de la planification familiale. (Rev Afr Santé Reprod 2004; 8[1]:57-62)

\section{Introduction}

Initiation of sexual behaviour is a normal part of human development and it often occurs during adolescence. This is common and universal to all societies and cultures around the world with implications for sexual and reproductive health. Adolescence is the period when much of the changes that are associated with becoming an adult take place.

Human development during adolescence is associated with consequences that require a public health response to them. The sheer number of adolescents, one in five persons (1.2 billion globally), with $86 \%$ living in Africa is a principal reason. In fact, in many countries of Africa, young people make up to $33 \%$ of the population. Another reason for a public health response relates to the fact that puberty with the attendant physical and psychological growth and maturation is an important milestone. The changes occurring during puberty are those societies expect and welcome. Fortunately, most adolescents go through these changes without significant problems. Nonetheless, all adolescents need support and care during this transition to adulthood, and some need special help.

Puberty is more than just a biological milestone. Emerging evidence indicates that puberty also has an association with a range of adolescent behaviours associated with adopting adult behaviours and emotional problems, independent of age of the adolescent. 1

In a public health response to the sexual and reproductive health of adolescents, the vision is to have healthy, competent, responsible adolescents with the capacity to form healthy social and sexual relationships, fulfil their sexual and reproductive aspirations, and who are able to 
contribute productively to the society. The goal is to promote healthy sexual development, reduce risk and vulnerability, and prevent morbidity and mortality associated with sexual activity and reproduction. ${ }^{2}$

It would be ideal to prevent the early initiation of sex, as there are specific vulnerabilities that accompany this. For example, there is the greater physiological vulnerability to reproductive tract infections including notably HIV, and the increased risks of mortality and morbidity associated with early pregnancy for the adolescent and her offspring. ${ }^{3}$ There are also social vulnerabilities; for example, the higher risk of sexual coercion due to their young age, social status, stigma and lost opportunities for personal and career development due to early pregnancy. When adolescents eventually become sexually active as most adults become, it is crucial that they have the information and the means to practice safe sex, so that sexual relations do not become a death sentence for them. Finally, it is important in a public health response to reduce the morbidity and mortality associated with sexual and reproductive activity, mainly unwanted pregnancies and reproductive tract infections.

Sexual and reproductive ill-health in adolescents is a big challenge. Of all the global burden of disease due to STIs, $19 \%$ is among adolescents, for maternal conditions (abortion, sepsis, haemorrhage, etc) $15 \%$ and for HIV $7 \%$. However, this does not tell the whole story. Data on new cases of HIV and STIs show that more than $50 \%$ of all new HIV infections are among young people, and the estimates for STIs are between 30 and $50 \%$. There are 69,000 maternal deaths annually in adolescents, which represents $13 \%$ of all maternal mortality. ${ }^{4}$ In the African region, according to data available to the WHO regional office for Africa, adolescents account for up to $40 \%$ of maternal mortality in some countries. ${ }^{5}$ Annually, an estimated 2-4.4 million adolescents resort to abortion worldwide. The most recent WHO estimates of unsafe abortion reveal that it is only in the African region that youths (15-24 years) account for more than $50 \%$ of all mortality (Figure 1). 6

Adolescents terminate unplanned pregnancies for several reasons. The reasons expose the relative lack of autonomy and low status of adolescents generally in the society. They also expose the relative lack of information for decision-making (about sexual activity and pregnancy) and services and supplies (for contraception) for adolescents that need them. They include the fear of expulsion from school, low income and other economic factors, lack of stable relationship, failed contraception due to poorer knowledge and access to services, being victim of rape or incest, not liking the father of the baby, and being forced to abort pregnancies through decisions made by others. ${ }^{7}$ There are several contributory factors to unsafe abortion in adolescents. The changing nature of the society, with attendant results on early sexual debut, poor access to contraception to prevent unwanted pregnancy, and inadequate access to safe abortion services (where not against the law), and the cost of such services (often available only in the private sector) are major contributors to unsafe abortion. There are also the issues of sexual abuse and coercion, which in many societies are often taboo to talk about. Another important contributory factor is a lack of, or poor quality, sexuality education. As well, many adolescents have poor knowledge of contraception.

The health-seeking behaviour of adolescents also differs in some significant respects to those of 
adults in the same predicament. A recent WHO review showed that adolescents are more likely than adults to delay seeking pregnancy care due to their relative inability to recognise pregnancy, admit being pregnant, or afford the cost of care, initially seeking help from unqualified sources. Other differences include resorting to unskilled providers, using dangerous methods to induce abortion such as ingesting toxic substances, inserting dangerous implements into the uterus. All these translate into the delay in presenting for care and a higher likelihood of complications due to delayed presentation. ${ }^{7}$

The consequences of unsafe abortion in adolescents are similar to those in adults. The medical consequences include genital lacerations, haemorrhage, perforation, sepsis, tetanus and some mental health problems such as depression. The difference is that the longer term effects of the complications can be more serious in younger persons than in adults. There are also more severe social consequences. For example, the effect of pelvic infection in a young person without previous children and infertility in Africa, where childlessness is viewed as very serious, is very different from that of a woman who already has children. Other social consequences may include stigma, which can negatively affect the chances of later marriage.

\section{Preventive Strategies}

In public health, it is the norm to discuss the prevention of health problems in terms of primary, secondary and tertiary prevention. For unsafe abortion, there is no doubt that preventing unwanted pregnancy is the key element of primary prevention. There are many African variations of the saying: "prevention is better than cure".

The WHO, in collaboration with UNFPA and UNICEF, developed a framework for programming for adolescents. ${ }^{8}$ The framework has at its core, promoting healthy development and building competencies of adolescents as being crucial to their ability to prevent and respond to health problems. The framework can be applied to the issue of unsafe abortion. The major interventions (Table 1) are strengthening health services and counselling, promoting a safe and supportive social environment in which adolescents can thrive and develop to their full potential, providing information and providing life and livelihood skills. The last three interventions (safe and supportive environment, information and skills) are key in primary prevention of unsafe abortion.

Secondary prevention would entail the prompt diagnosis and management of complications. There are several health systems issues that relate to secondary prevention. Health workers are often not trained or are not prepared to work with adolescents, and referral pathways are often weak especially between public health services and other sectors (such as private formal and non-formal practitioners) where more adolescents are likely to seek help. Besides, there are legal and policy barriers to the provision of services to adolescents in many countries.

There are recent efforts to improve the health sector response to a variety of health issues especially with regards to making health services more responsive to the needs of adolescents (Figure 2). ${ }^{9}$ The issues listed under attributes are important for all women seeking care, but they are especially important for adolescents who are usually reluctant to seek care. These include 
improving the attitude and competence of health workers, protecting confidentiality, ensuring affordability, and promoting appropriate and safe use of technology. Concerning the content of preventive and curative care, commodities for the practice of safer sex for the prevention of pregnancy and infections are important for sexually active adolescents. Treatment of complications of unsafe abortion is the same in adolescents as for adults. However, health workers need to overcome their possible discomfort with adolescent sexuality, particularly of unmarried adolescents. They also need appropriate skills to handle adolescents including appropriate history-taking and counselling skills. ${ }^{10}$

Post-abortion care to adolescents is also import-ant. There are some likely differences in content when compared with adult care particularly through advice on schooling, relationships and sexuality issues, and adherence to follow-up and continuity of care. This is all because health-seeking is a learned behaviour, and there is presumably a relative lack of experience of adolescents in interaction with the heath care system and other relationship issues.

\section{Controversies}

A controversial primary prevention intervention area is the provision of sexuality education, and the concern that this may lead to increased sexual activity in adolescents. A major review by UNAIDS, in addition to more recent reviews by the FOCUS project, as well as most recent WHO reviews on effectiveness of several interventions for adolescent sexual and reproductive health, found that education on sexual health and/or HIV does not encourage increased sexual activity. Indeed, this may help delay the first sexual intercourse, and protect sexually active youths from STIs including HIV and pregnancy. ${ }^{11}$ The studies showed that responsible and safe behaviour can be learned. They recommended that sexual health education is best started before the onset of sexual activity. ${ }^{11,12}$ Young people are heterogeneous and cannot all be reached by the same techniques, besides, it would make better sense that adequate information should precede decision-making.

It is also pertinent to examine the issue of absti-nence as part of sexuality education. It is important to promote voluntary abstinence among young people as the only $100 \%$ effective way of avoiding the negative consequences of sex. However, the evidence is ample that many young people in Africa are having sex sometimes for pleasure, to achieve intimacy and develop relationships. Many of them also have sex frequently as a response to the emptiness and hopelessness of their lives, or because of the social, economic and cultural pressures that incite them to say "yes" when they would prefer to say "no". More research needs to be done to identify clearly the role of "abstinence-only" programmes. Currently available evidence indicates that programmes that present abstinence to young people together with information about safer sex practices, such as condoms, are more effective. ${ }^{13}$ Therefore, while efforts continue on many fronts such as (a) developing sustainable and effective "abstinence-only" interventions; and (b) alleviating the factors in the social environment that incite young people into having sex (which are long-term efforts), the public health (and human rights) challenge is to ensure that young people who do engage in sex have access to those evidence-based interventions that help to ensure that it does not end in disease or death for themselves or for their partners. 
Another intervention area in the primary prevention of unsafe abortion in adolescents relates to the creation of safe and supportive environment for adolescents to live and thrive. ${ }^{8}$ Adolescents live in a world shaped by adults. The laws, policies and social norms are set by adults. In the African region, for example, there is evidence of inter-generational sex contributing to the HIV epidemic. Coercion and rape are realities of adolescent sexuality in Africa. A recent WHO review of data from 57 countries from all regions of the world suggests that certain protective and risk factors can explain the differences in adolescent health risk behaviours and health outcomes, even after accounting for age, socio-economic status, sex and ethnic group differences. ${ }^{14}$ Early sexual initiation, which is associated with unprotected sexual intercourse and its consequences, was found to be associated with relationships with parents, schools, friends, beliefs and other risk behaviours of the adolescent. Adolescents who have positive relationships with parents and teachers, and who have spiritual beliefs, are less likely to initiate sexual intercourse early. Those who believe that their friends are sexually active and who engage in other risk behaviours such as alcohol and drugs are more likely to initiate sexual intercourse early (Table 2). ${ }^{14}$

In summary, it is important to note that adole-scence is a time of risks and opportunities as described in this paper. Risks include unsafe sex and unwanted pregnancy with the resultant unsafe abortions. Opportunities abound for providing information and education to adolescents, which will shape their lives and those of future generations. Finally, the director-general of the WHO once said that young people need adult assistance to deal with thoughts, feelings and experiences that accompany physical maturity. By providing this help, we are not encouraging irresponsible lifestyles. Evidence from around the world has clearly shown that providing information and skills on human sexuality and human relationships helps avert health problems and creates more mature and responsible attitudes. ${ }^{15}$

\section{Acknowledgements}

We gratefully acknowledge the useful comments of Dr Tomris Turmen and Ms Jane Cottingham on the presentation that resulted in this paper, and the comments of Jane Ferguson, all of the WHO, Geneva.

\section{References}

1. Patton George. Presentation to the WHO technical meeting on the very young adolescents, Geneva, April 2003.

2. World Health Organization. Information brief on adolescent sexual and reproductive health. Unpublished, 2002.

3. Treffers PE, Olukoya AA, Ferguson BJ and Liljestrand J. Care for adolescent pregnancy and child birth. Int J Gynaecol Obstet 2001; 75: 111-121.

4. World Health Organization. Global Programme on Evidence. Geneva, 2000.

5. World Health Organization, Regional Office for Africa. Adolescent Health: A Strategy for the African Region. AFR/RC51/10 Rev.1, 2001. 
6. Åhman E and Shah I. Unsafe abortion: worldwide estimates for 2000. Reprod Health Matters 2002; 10(19): 13-17.

7. Olukoya AA, Kaya, AA, Ferguson BJ and AbouZahr C. Unsafe abortion in adolescents. Int J Gynaecol Obstet 2001; 75: 137-147.

8. World Health Organization. Programming for adolescent health and development. WHO Technical Report Series 886, Geneva, 1999.

9. World Health Organization. Adolescent Friendly Health Services: An Agenda for Change. WHO/FCH/CAH/02.14, 2002.

10. World Health Organization. Safe Abortion: Technical and Policy Guidance for Health Systems. Geneva, 2003.

11. UNAIDS. Impact of HIV and Sexual Health Education on the Sexual Behaviour of Young People: A Review Update. UNAIDS/97.4, Geneva, 1997.

12. Pathfinder International. FOCUS for young adults, advancing young adult reproductive health: actions for the next decade. End of project report. Washington DC, 2001.

13. World Health Organization, Department of Child and Adolescent Health and Development. Briefing notes on selected adolescent health issues: abstinence. Unpublished draft, 2001.

14. World Health Organization. Broadening the Horizon: Balancing Protection and Risk for Adolescents. WHO/FCH/CAH/01.20, Geneva, 2001.

15. World Health Organization. Dr Gro Harlem Brundt-land, Ministerial Conference on Population and Development, The Hague, February 1999.

(C) Women's Health and Action Research Centre 2004

\section{The following images related to this document are available:}

\section{Photo images}

[rh04010f1.jpg] [rh04010t1.jpg] [rh04010f2.jpg] [rh04010t2.jpg] 


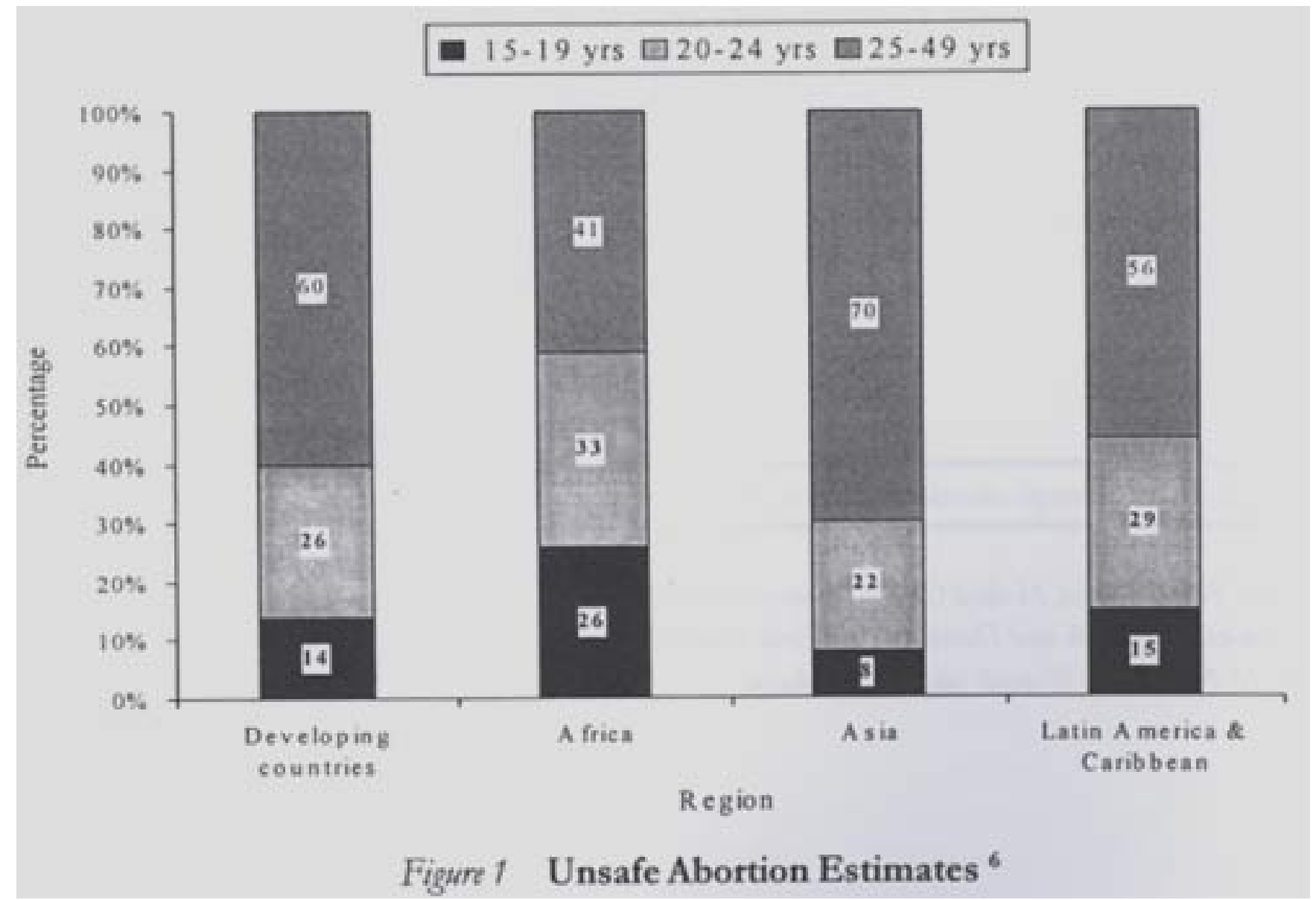




\section{Table 1 Major Interventions for Adolescent Health and Development ${ }^{8}$}

1. Create safe and supportive environment

2. Provide information

3. Build skills

4. Provide counselling

5. Improve health services 


\section{PROVIDING HEALTH SERVICES AND COUNSELLING}

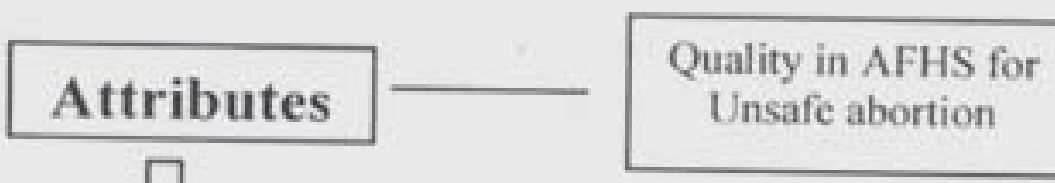

- Non-judgemental

- Confidential

- Appropriate \& safe technology

- Competency of health workers

4. Affordable

Figure 2 Some Attributes of Health Services that are Responsive to Adolescents' Needs for the Prevention of Unsafe Abortions? 


\section{Table 2 Risk and Protective Factors for Early Sexual Initiation ${ }^{4}$}

\begin{tabular}{llccc} 
Risk and protective factors & Africa & Asia & Caribbean & $\begin{array}{c}\text { South } \\
\text { America America }\end{array}$ \\
\hline
\end{tabular}

A positive relationship with parents A positive relationship with teachers Friends who are sexually active Engaging in other risky behaviours Having spiritual beliefs

\begin{tabular}{|c|c|}
\hline a & • \\
\hline$\bullet$ & (1) \\
\hline $\boldsymbol{\Delta}$ & E \\
\hline $\mathbf{\Delta}$ & ㅁ \\
\hline ㅁ & - \\
\hline
\end{tabular}

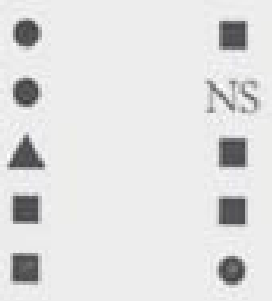
- = Protective factor
$\boldsymbol{\Delta}=$ Risk factor
= Not measured
NS $=$ Not significant 